\title{
Assessment of Microhardness Profile in Grinding Using Barkhausen Noise Technique at Various Analysis Parameters
}

\author{
Saurabh Kumar, Mukesh Yadav, Prateek Agrawal, Mohd Zaheer Khan, \\ and Meghanshu Vashista \\ Department of Mechanical Engineering, Institute of Technology, Banaras Hindu University, Varanasi, Uttar Pradesh 221005, India \\ Correspondence should be addressed to Meghanshu Vashista, mvashista@gmail.com
}

Received 7 April 2011; Accepted 16 May 2011

Academic Editor: S. J. Milne

Copyright $\odot 2011$ Saurabh Kumar et al. This is an open access article distributed under the Creative Commons Attribution License, which permits unrestricted use, distribution, and reproduction in any medium, provided the original work is properly cited.

\begin{abstract}
An experimental investigation was carried out to study the effect of variation in Barkhausen noise (BN) analysis parameters (magnetizing voltage and excitation frequency) for assessment of microhardness profile upon surface grinding. A varying amount of surface damage was induced in the ground samples by changing the downfeed during grinding. It was observed that variation in microhardness inversely affects root mean square (rms) value of BN signal. The observed BN profile indicated no phase transformation or microstructural changes upon grinding. Metallographic study of ground samples has been undertaken to confirm the results obtained by BN analysis. Barkhausen noise parameter (rms value of BN signal), at elevated magnetizing voltage and excitation frequency, increased with reduction in microhardness along the depth indicating the applicability of BN analysis technique in assessing the microhardness profile of ground steel.
\end{abstract}

\section{Introduction}

Barkhausen Noise Analysis (BNA) technique is a nondestructive testing (NDT) method for evaluation of surface integrity in ferromagnetic steels. BNA technique offers certain advantages such as the greater depth of penetration, faster measurement, portability of equipment, and capability to measure components having complex geometries like gears, over other NDT techniques, thus making this as a potential NDT technique for assessing the surface integrity upon grinding [1-3]. The maximum depth of $\mathrm{BN}$ signal that can be detected on the surface (skin depth) poses a limitation for evaluation of variation in surface integrity aspects in the depth direction.

The maximum depth from which $\mathrm{BN}$ signal is detected (skin depth) depends on magnetic field strength and its excitation frequency, permeability of the medium, distance between the poles of the electromagnetic yoke, sensitivity of the BN signal, and so forth [4]. Excitation frequency and magnetizing voltage are important measurement parameters of BNA technique to access information from various penetration depths [5]. Increase in excitation frequency of magnetic field can increase depth of measurement $[6,7]$. Magnetizing voltage applied to the magnetizing coil affects the penetration depth [8]. Augustyniak et al. [9] analyzed the impact of variation in magnetizing frequency over $\mathrm{BN}$ amplitude while evaluating the time and space distribution of magnetic flux density. Mayos et al. [10-12] investigated changes in magnetic properties of Barkhausen emission at different frequencies while assessing surface decarburization in steels at different depths.

Microhardness profile is an important surface integrity characteristic as it may alter the surface properties. Grinding is, typically, last tooling operation which decides the surface integrity of component; however, grinding causes variation in microhardness along the depth of ground sample. Assessment of microhardness profile of ground samples using conventional technique is quite often time consuming. Literature survey indicates that BNA technique has been successfully attempted for surface hardness assessment at constant analysis parameters (i.e., magnetizing voltage and excitation frequency). Hence, in the present study an attempt 
has been made to analyze the effect of varying magnetizing frequency and magnetizing voltage for assessment of microhardness profile upon grinding mild steel sample.

\section{Experimental}

Grinding tests were undertaken on mild steel with nominal hardness of $320 \mathrm{HV}_{0.05}$. The chemical composition of mild steel is given in Table 1 .

Grinding experiments were conducted with rectangular shape specimens $\left[75^{L} \times 10^{H} \times 9^{W} \mathrm{~mm}\right]$ in plunge surface grinding mode using an Alex Surface Grinder with six levels of downfeed from $10 \mu \mathrm{m}$ to $60 \mu \mathrm{m}$. Table 2 shows the grinding process details.

Ground samples were sectioned perpendicular to the grinding direction for preparing of micrograph. These were subsequently hot-moulded in specimen mount press (Buehler, USA) by phenolic resin powder, along with dummy samples for maximum edge retention. Moulded samples were polished on different emery papers with successively fine grit of mesh of 220, 400, and 600 followed by cloth polishing with $\mathrm{Al}_{2} \mathrm{O}_{3}$ paste and finally diamond-polished on auto-disc polishing machine (Struers LaboForce-3) until a mirror like surface was obtained, cleaning using lab detergent, and then etching for about 10-seconds using $5 \%$ nital solution. The samples were immediately rinsed using running water and dried using hot air for observation of cross-section of the ground surface under an inverted metallurgical optical microscope (Olympus, Japan). Microhardness tester LM 700 (Leco, USA) was employed to study the variation in the microhardness (HV) of ground samples along the depth. Microhardness measurements were carried out with $50 \mathrm{gm}$ load and 10-second dwell time on the crosssections of ground samples with a Vickers indenter starting from $25 \mu \mathrm{m}$ depth from the surface. This is a practical limitation because of loss of material constraint at positions closer to the surface, and a limit of 2.5 times the indentation diagonal as recommended by ASTM E 92 standard. Each data point represents average of three measurements.

The BN measurements were taken using a commercially available $\mu$ scan/Rollscan-300 system supplied by Stresstech, Finland. A flat surface probe, with a BN signal pick-up coil at the centre, was used to apply the excitation magnetic field and to receive the $\mathrm{BN}$ signal. Ground samples were cleaned before measurement by isopropyl in order not to affect the BN signal which is surface dependent. The central area of samples was taken as the location for $\mathrm{BN}$ measurement. Among the different spectrum analysis parameters; rms value and peak profile of $\mathrm{BN}$ signal were selected for analysis. $\mathrm{BN}$ measurements were carried out at various excitation frequencies and magnetizing voltage. Table 3 represents the summary of analysis parameters used in Barkhausen noise analysis.

\section{Results and Discussions}

3.1. Metallography Analysis. Grinding wheel acts as a heat source during the grinding process. It is likely that

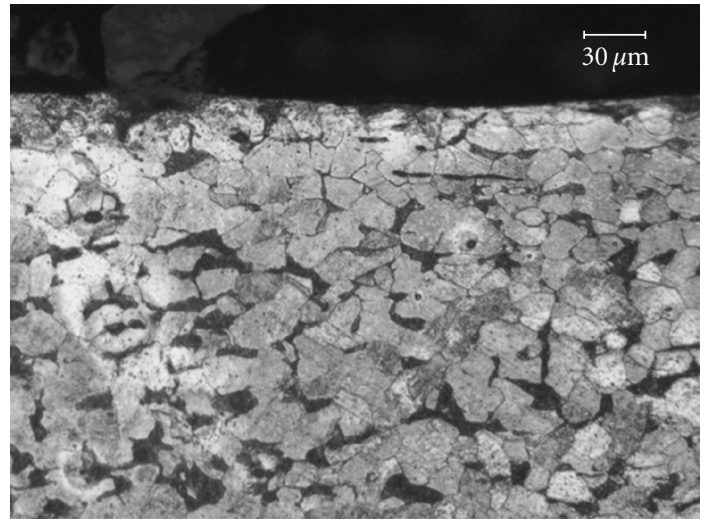

FIgURE 1: Micrograph of ground sample at $10 \mu \mathrm{m}$ downfeed.

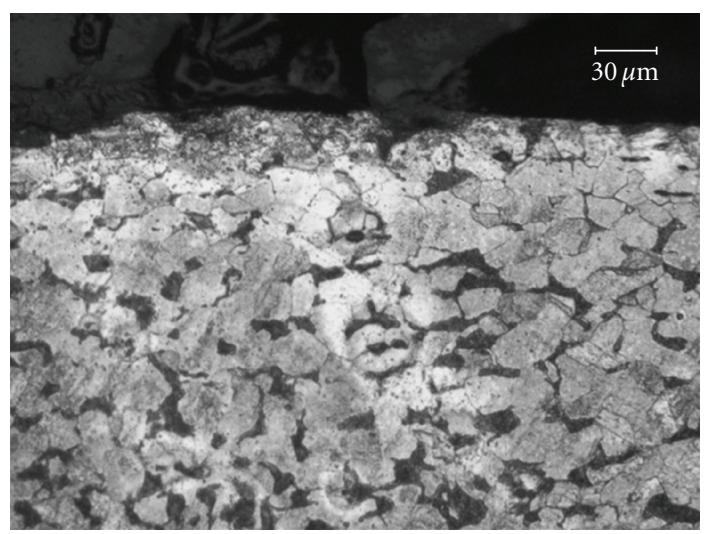

FIgURE 2: Micrograph of ground sample at $60 \mu \mathrm{m}$ downfeed.

microstructural changes may occur below the surface due to immediate cooling by coolant and bulk material during grinding operation.

Moorthy et al. [1] pointed out that thermoplastic deformation during grinding results in the alteration of microstructure, such as tempering of martensite and conversion of retained austenite to martensite in the near-surface layers. These metallurgical transformations also affect $\mathrm{BN}$ profile. Eda et al. [13] and Nguyen et al. [14] observed phase transformation in medium carbon steel samples upon grinding with alumina grinding wheel. On the other hand, Vashista et al. [2] and Sosa et al. [15] did not observed any phase transformation in grinding.

Metallography analysis was carried out to reveal phase transformation, but no significant phase transformation was visible throughout the experimental domain. Figures 1 and 2 exhibit micrographs of cross-section of ground samples at different downfeeds $10 \mu \mathrm{m}$ and $60 \mu \mathrm{m}$, respectively.

Figure 2 clearly reveals that no microstructrual changes or phase transformation took place upon grinding even at higher level of downfeed. Similar results in the metallorgaphy analysis were also reported by many researchers upon grinding various materials $[2,16,17]$. 
TABLe 1: Chemical composition of mild steel.

\begin{tabular}{lccccccc}
\hline Chemical element & Fe & C & Si & Mn & Cr & P & S \\
\hline$\%$ & Balance & 0.30 & 0.1 & 0.49 & 0.04 & 0.03 & 0.04 \\
\hline
\end{tabular}

TABLE 2: Experimental conditions for conventional grinding of mild steel.

\begin{tabular}{lc}
\hline Grinding machine & Alex surface grinder \\
Wheel velocity $(\mathrm{m} / \mathrm{s})$ & 31.4 \\
Grinding wheel & Abrasive- $\mathrm{Al}_{2} \mathrm{O}_{3}$ \\
& Mesh size-60 \\
Workpiece & Mild steel \\
Environment & Wet, $1: 20$ soluble oil \\
Work velocity $(\mathrm{m} / \mathrm{min})$ & 8 \\
Grinding mode & Up grinding \\
Downfeed $(\mu \mathrm{m})$ & $10,20,30,40,50$, and 60 \\
\hline
\end{tabular}

TABLE 3: Parameters used in BN analysis.

\begin{tabular}{lc}
\hline Magnetizing voltage (volt) & $3.5,4.0,4.5$, and 5.0 \\
Magnetizing frequency $(\mathrm{Hz})$ & 75 and $100 \mathrm{~Hz}$ \\
Filter & $70-200 \mathrm{kHz}$ \\
Number of burst & 4 \\
\hline
\end{tabular}

3.2. Microhardness. Grinding operation generates excessive heat in grinding zone that leads to elevated temperature condition. This elevated temperature condition followed by rapid cooling action of coolant induces plastic deformation at various degrees along the depth of ground surface. Induction of varying degree of plastic deformation affects the microhardness profile of ground surface. Moorthy et al. [1] plotted microhardness profile of case carburized En36 steel after finish grinding and observed decrease in microhardness along the depth below the ground surface. Similarly, Vashista et al. $[2,3]$ and Vashista and Paul [18-20] also observed reduction in microhardness along the depth below ground surface while grinding medium carbon steel samples using various variant of grinding process. Figures 3 and 4 show variation in microhardness of the ground sample along the depth from the edge of the ground surface for different downfeed undertaken.

Typically, Vickers hardness increases with plastic deformation [21-23]. Thompson and Tanner [24] studied the effect of plastic deformation on Vickers hardness value and observed that Vickers hardness increases with plastic deformation when pearlitic steel bars were deformed to different degrees of tensile strain. Grinding condition with higher chip thickness and chip load is known to generate higher plastic deformation on surface and subsurface layer, thus leading to work hardening [25]. The plastic deformation generated in the grinding process also led to slight increase in microhardness near the ground surface due to process hardening effect [26].

The microhardness did not show any significant variation along the depth for the sample ground at downfeed of

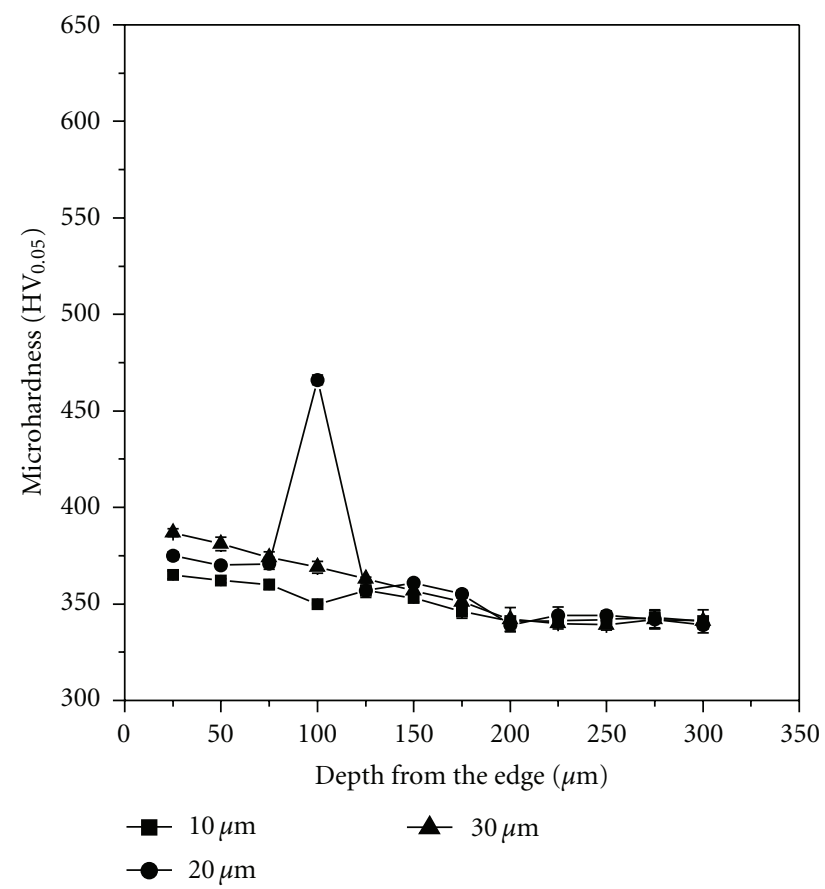

FIgURE 3: Variation in microhardness along the depth at various downfeeds (10 to $30 \mu \mathrm{m}$ ).

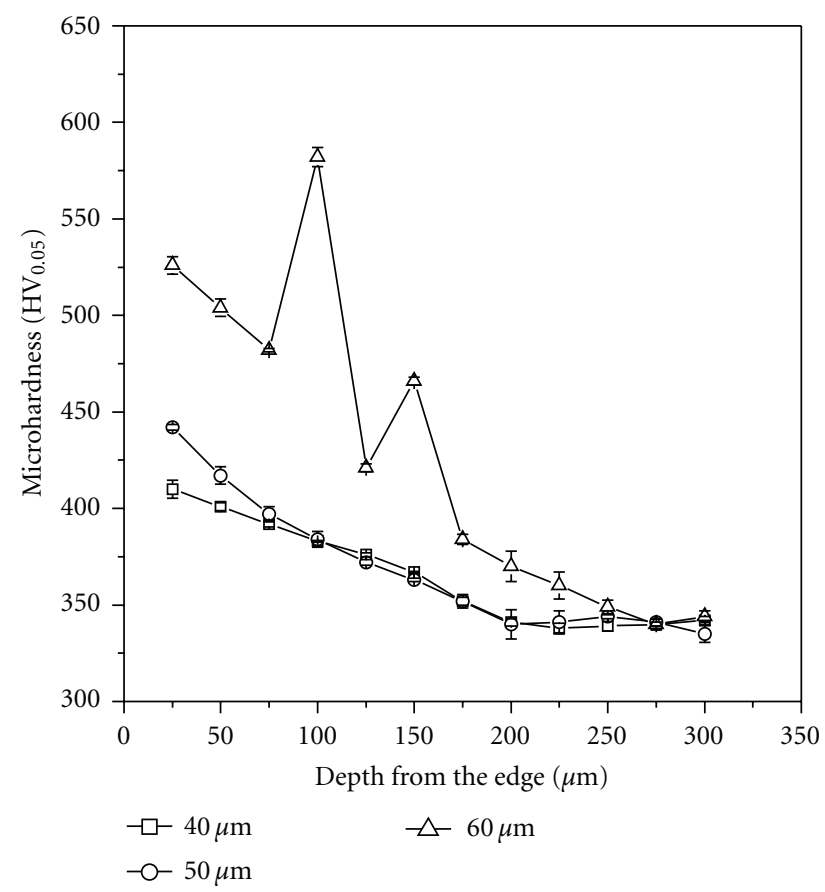

FIGURE 4: Variation in microhardness along the depth at various downfeed (40 to $60 \mu \mathrm{m}$ ). 


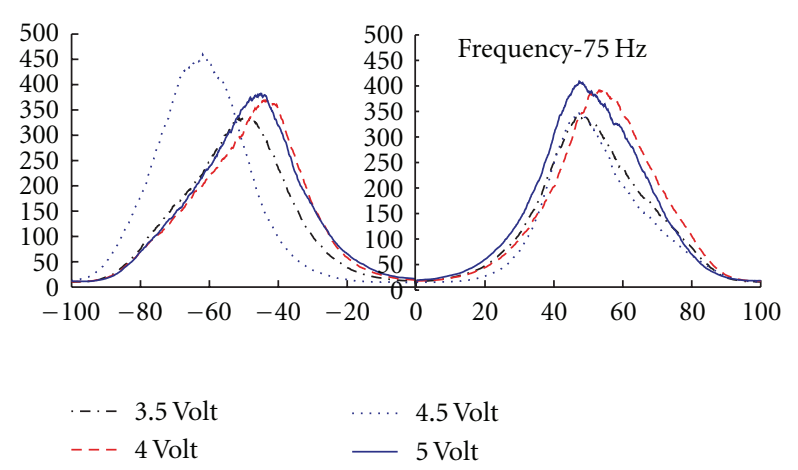

Figure 5: BN profiles at excitation frequency $75 \mathrm{~Hz}$ and various magnetizing voltage.

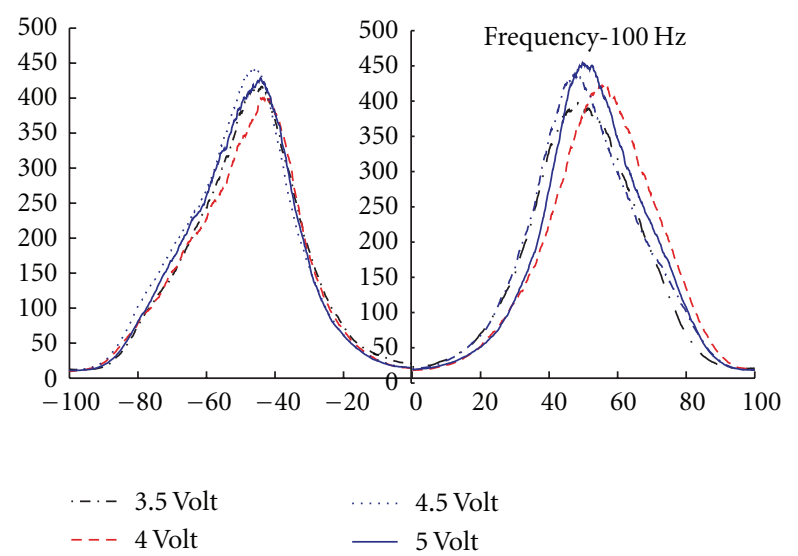

FIgURe 6: BN profiles at excitation frequency $100 \mathrm{~Hz}$ and various magnetizing voltage.

$10 \mu \mathrm{m}$ as shown in Figure 3. However, microhardness near the ground surface increased appreciably with downfeed as represented in Figures 3 and 4. Microhardness gradually decreased along the depth below the ground surface. Such increase in microhardness at the surface typically occurs due to grain refinement, phase transformation, and plastic deformation as reported earlier [13-15, 27, 28].

\subsection{Barkhausen Analysis}

3.3.1. BN Profile. Typically BN profiles contain one or more peaks. Thompson and Tanner [29] reported that BN profile of ferritic-pearlitic steel contained a single peak when volume fraction of pearlite was high, and that profile evolved into a double-peak profile as the volume fraction of ferrite increased. Moorthy et al. [30] observed that BN profile systematically changes from single peak to two peaks with increase in tempering time while tempering quenched ferritic steel. Figures 5 and 6 exhibit the BN profiles (at various analysis parameters) obtained from ground sample upon grinding at $60 \mu \mathrm{m}$ downfeed.

The BN profiles obtained from the mild steel sample ground at lower downfeed $(10 \mu \mathrm{m})$ and higher downfeed $(60 \mu \mathrm{m})$ contain single peak. This can be attributed to the fact that during the present study, microstructural change or phase transformation was absent upon grinding as revealed by metallography analysis. Nevertheless, the amplitudes of the present profiles are not identical, as variation of microhardness in these ground samples has induced $\mathrm{BN}$ signal of different intensities.

3.3.2. BN Signal (rms Value). Thermoplastic deformation during the abusive grinding process results in alteration in microhardness along the depth owing to plastic deformation, as earlier presented in microhardness analysis. A handful of authors have analyzed the effect of change in surface hardness on BN parameters. Mészáros [21] observed linear proportionality between inverse of $\mathrm{BN}$ (rms) and microhardness, while characterizing plastically strained ferritic stainless steel samples. O'Sullivan et al. [22] observed inverse correlation between microhardness and $\mathrm{BN}$ (rms) while characterising ferritic stainless steel sample plastically deformed to various degrees. They also advocated use of micromagnetic technique for assessment of microhardness. In another study, O'Sullivan et al. [23] observed decrease in BN (rms) with increase in microhardness while characterizing plastically deformed and heat treated AISI 430 ferritic stainless steel samples. Hardness increases with plastic deformation due to the increased dislocation density, which impedes magnetic domain walls movement as the magnetizing force is not enough to set the domain walls free, and reduced domain movement results in decreased BN activity. Figures 7 and 8 show variation in $\mathrm{BN}$ (rms) for ground samples (downfeed 10 to $60 \mu \mathrm{m}$ ) at various Barkhausen noise analysis parameters.

Increase in excitation frequency and magnetizing voltage can increase depth of penetration, as reported earlier. During present study, microhardness analysis along the depth indicates reduction in microhardness below the ground surface, throughout the experiment domain, without any microstructural changes. As reduction in microhardness increases the BN intensity level, thus, increase in BN parameter with analyzing parameters (magnetizing frequency and voltage as represented in Figures 7 and 8) can be attributed to reduction in microhardness along the depth, as clearly revealed in microhardness profiles of ground samples.

\section{Conclusions}

(a) $\mathrm{BN}$ signal was observed to vary with excitation frequency and magnetizing voltage.

(b) No microstructural changes/phase transformation was observed throughout the grinding domain.

(c) Single peak BN profile obtained throughout the experimental domain supported no appreciable microstructural changes or phase transformation as revealed by metallographic analysis with increase in downfeed.

(d) Microhardness was found to increase with downfeed due to associated plastic deformation, and along 


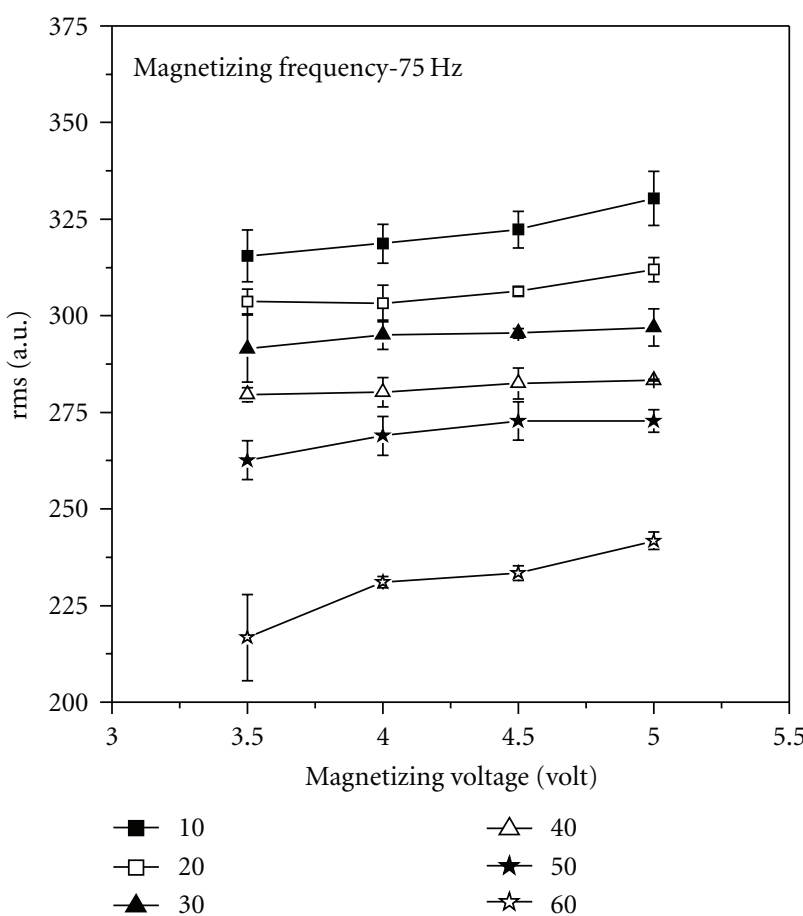

FIGURE 7: Variation in BN (rms) with downfeed and magnetizing voltage at magnetizing frequency $75 \mathrm{~Hz}$.

the depth microhardness reduced continuously up to $300 \mu \mathrm{m}$ depth below the surface.

(e) Intensity of BN signal increases with reduction in microhardness.

(f) Increase in BN (rms) value at elevated magnetizing voltage and excitation frequency, with reduction in microhardness along the depth, demonstrated the applicability of BNA technique in assessing the microhardness profile of ground steel.

\section{Acknowledgment}

The authors gratefully acknowledge Professor S. Paul, Mechanical Engineering Department, Indian Institute of Technology Kharagpur, Kharagpur (India) for providing the facility to conduct the grinding experiments and analyzing the ground samples.

\section{References}

[1] V. Moorthy, B. A. Shaw, P. Mountford, and P. Hopkins, "Magnetic Barkhausen emission technique for evaluation of residual stress alteration by grinding in case-carburised En36 steel," Acta Materialia, vol. 53, no. 19, pp. 4997-5006, 2005.

[2] M. Vashista, S. Ghosh, and S. Paul, "Application of micromagnetic technique in surface grinding for assessment of surface integrity," Materials and Manufacturing Processes, vol. 24, no. 4, pp. 488-496, 2009.

[3] M. Vashista, S. Kumar, A. Ghosh, and S. Paul, "Surface integrity in grinding medium carbon steel with miniature electroplated monolayer cBN wheel," Journal of Materials

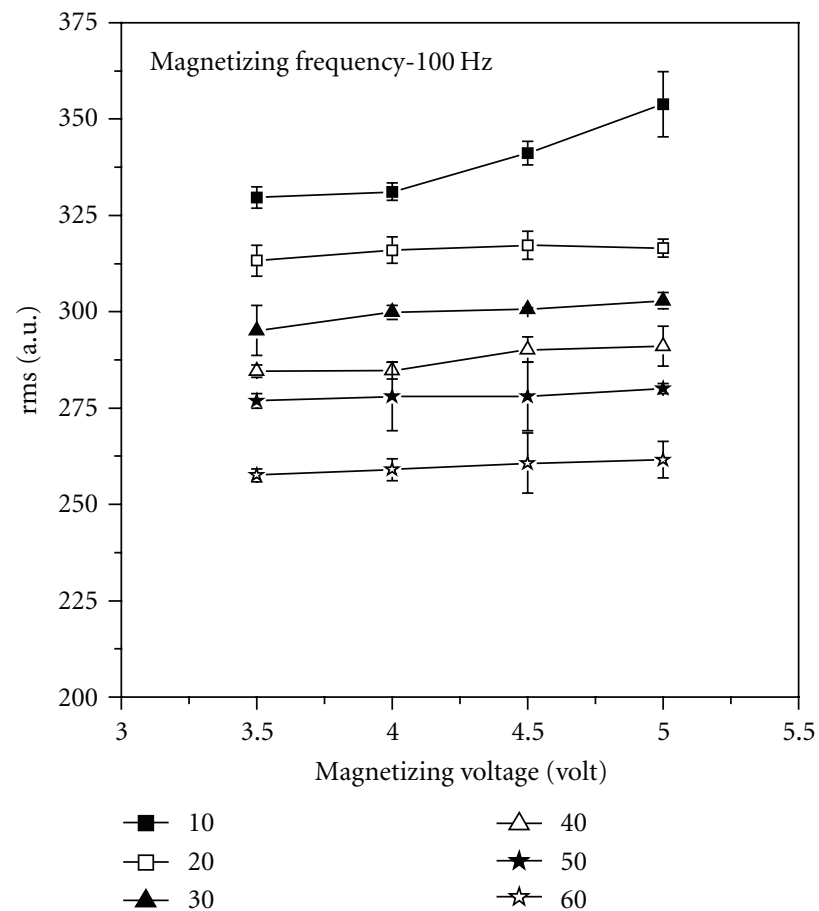

FIGURE 8: Variation in BN (rms) with downfeed magnetizing voltage at magnetizing frequency $100 \mathrm{~Hz}$.

Engineering and Performance, vol. 19, no. 9, pp. 1248-1255, 2010.

[4] V. Moorthy, S. Vaidyanathan, T. Jayakumar, and R. Baldev, "Microstructural characterization of quenched and tempered $0.2 \%$ carbon steel using magnetic Barkhausen noise analysis," Journal of Magnetism and Magnetic Materials, vol. 171, no. 1-2, pp. 179-189, 1997.

[5] A. Hug, X. Kleber, J. Merlin, and H. Petitgand, "Use of magnetic Barkhausen noise measurements for multi-phase steels characterization," in Proceedings of the 5th International Conference on Barkhausen Noise and Micromagnetic Testing, pp. 37-45, 2005.

[6] S. F. Silva Jr. and T. R. Mansur, "Determining residual stresses in ferromagnetic materials by Barkhausen noise measurement," in Proceedings of the of 15th World Conference on NonDestructive Testing, Rome, Italy, 2000.

[7] J. M. Makar and B. K. Tanner, "Effect of plastic deformation and residual stress on the permeability and magnetostriction of steels," Journal of Magnetism and Magnetic Materials, vol. 222, no. 3, pp. 291-304, 2000.

[8] I. S. Harrison, Detecting white layer in hard turned components using Non-destructive methods, M.S. thesis, Georgia Institute of Technology, 2004.

[9] M. Augustyniak, B. Augustyniak, L. Piotrowski, M. Chmielewski, and M. Sablik, "Evaluation of time and space distribution of magnetic flux density in a steel plate magnetized by a C-Core," in Proceedings of the of 5th International Conference on Barkhausen Noise and Magnetic Testing, pp. 191-198, 2005.

[10] M. Mayos, "Non-destructive testing of surface decarburization," Report No. 1222, French Iron and research Institute (IRSID), 1985. 
[11] M. Mayos, M. Putignani, and S. Segalini, "Nondestructive testing of surface decarburization of steel," Report No. 1203, French Iron and Research Institute (IRSID), 1985.

[12] M. Mayos, S. Sagalini, and M. Putignani, "Electro-magnetic non-destructive evaluation of surface decarburization on steels: feasibility and possible applications," Review of Progress in Quantitative NDE, 1987.

[13] H. Eda, E. Ohmura, S. Yamauchi, and I. Inasaki, "Computer visual simulation on structural changes of steel in grinding process and experimental verification," CIRP Annals, vol. 42, no. 1, pp. 389-392, 1993.

[14] T. Nguyen, I. Zarudi, and L. C. Zhang, "Grinding-hardening with liquid nitrogen: mechanisms and technology," International Journal of Machine Tools and Manufacture, vol. 47, no. 1, pp. 97-106, 2007.

[15] A. D. Sosa, M. D. Echeverría, O. J. Moncada, and J. A. Sikora, "Residual stresses, distortion and surface roughness produced by grinding thin wall ductile iron plates," International Journal of Machine Tools and Manufacture, vol. 47, no. 2, pp. 229-235, 2007.

[16] M. J. Balart, A. Bouzina, L. Edwards, and M. E. Fitzpatrick, "The onset of tensile residual stresses in grinding of hardened steels," Materials Science and Engineering A, vol. 367, no. 1-2, pp. 132-142, 2004.

[17] L. R. da Silva, E. C. Bianchi, R. Y. Fusse, R. E. Catai, T. V. França, and P. R. Aguiar, "Analysis of surface integrity for minimum quantity lubricant-MQL in grinding," International Journal of Machine Tools and Manufacture, vol. 47, no. 2, pp. 412-418, 2007.

[18] M. Vashista and S. Paul, "Study of the effect of process parameters in high-speed grinding on surface integrity by Barkhausen noise analysis," Proceedings of the Institution of Mechanical Engineers, Part B, vol. 222, no. 12, pp. 1625-1637, 2008.

[19] M. Vashista and S. Paul, "Correlation between surface integrity of ground medium carbon steel with Barkhausen Noise parameters and magnetic hysteresis loop characteristics," Materials and Design, vol. 30, no. 5, pp. 1595-1603, 2009.

[20] M. Vashista and S. Paul, "On the critical behaviour of Barkhausen noise parameters in surface grinding," International Journal of Abrasive Technology, vol. 2, no. 2, pp. 184-206, 2009.

[21] I. Mészáros, "Complex magnetic investigation of ferritic stainless steel," Materials Science Forum, vol. 473-474, pp. 231236, 2005.

[22] D. O’Sullivan, M. Cotterell, D. A. Tanner, and I. Mészáros, "Characterisation of ferritic stainless steel by Barkhausen techniques," NDT and E International, vol. 37, no. 6, pp. 489496, 2004.

[23] D. O’Sullivan, M. Cotterell, S. Cassidy, D. A. Tanner, and I. Mészáros, "Magneto-acoustic emission for the characterisation of ferritic stainless steel microstructural state," Journal of Magnetism and Magnetic Materials, vol. 271, no. 2-3, pp. 381389, 2004.

[24] S. M. Thompson and B. K. Tanner, "The magnetic properties of specially prepared pearlitic steels of varying carbon content as a function of plastic deformation," Journal of Magnetism and Magnetic Materials, vol. 132, no. 1-3, pp. 71-88, 1994.

[25] A. D. Sosa, M. D. Echeverría, O. J. Moncada, and S. N. Simison, "Surface reactivity of thin wall ferritic ductile iron. The effect of nodule count and grinding variables," Materials Letters, vol. 62, no. 1, pp. 100-102, 2008.
[26] X. P. Xu, Y. Q. Yu, and H. J. Xu, "Effect of grinding temperatures on the surface integrity of a nickel-based superalloy," Journal of Materials Processing Technology, vol. 129, no. 1-3, pp. 359-363, 2002.

[27] T. Matsuo, H. Shibahara, and Y. Ohbuchi, "Curvature in surface grinding of thin workpieces with superabrasive wheels," CIRP Annals, vol. 36, no. 1, pp. 231-234, 1987.

[28] Y. Ohbuchi and T. Matsuo, "Force and chip formation in single-grit orthogonal cutting with shaped $\mathrm{cBN}$ and diamond grains," CIRP Annals, vol. 40, no. 1, pp. 327-330, 1991.

[29] S. M. Thompson and B. K. Tanner, "The magnetic properties of pearlitic steels as a function of carbon content," Journal of Magnetism and Magnetic Materials, vol. 123, no. 3, pp. 283298, 1993.

[30] V. Moorthy, S. Vaidyanathan, T. Jayakumar, B. Raj, and B. P. Kashyap, "Effect of tensile deformation on micromagnetic parameters in $0.2 \%$ carbon steel and $2.25 \mathrm{Cr}-1 \mathrm{Mo}$ steel," Acta Materialia, vol. 47, no. 6, pp. 1869-1878, 1999. 

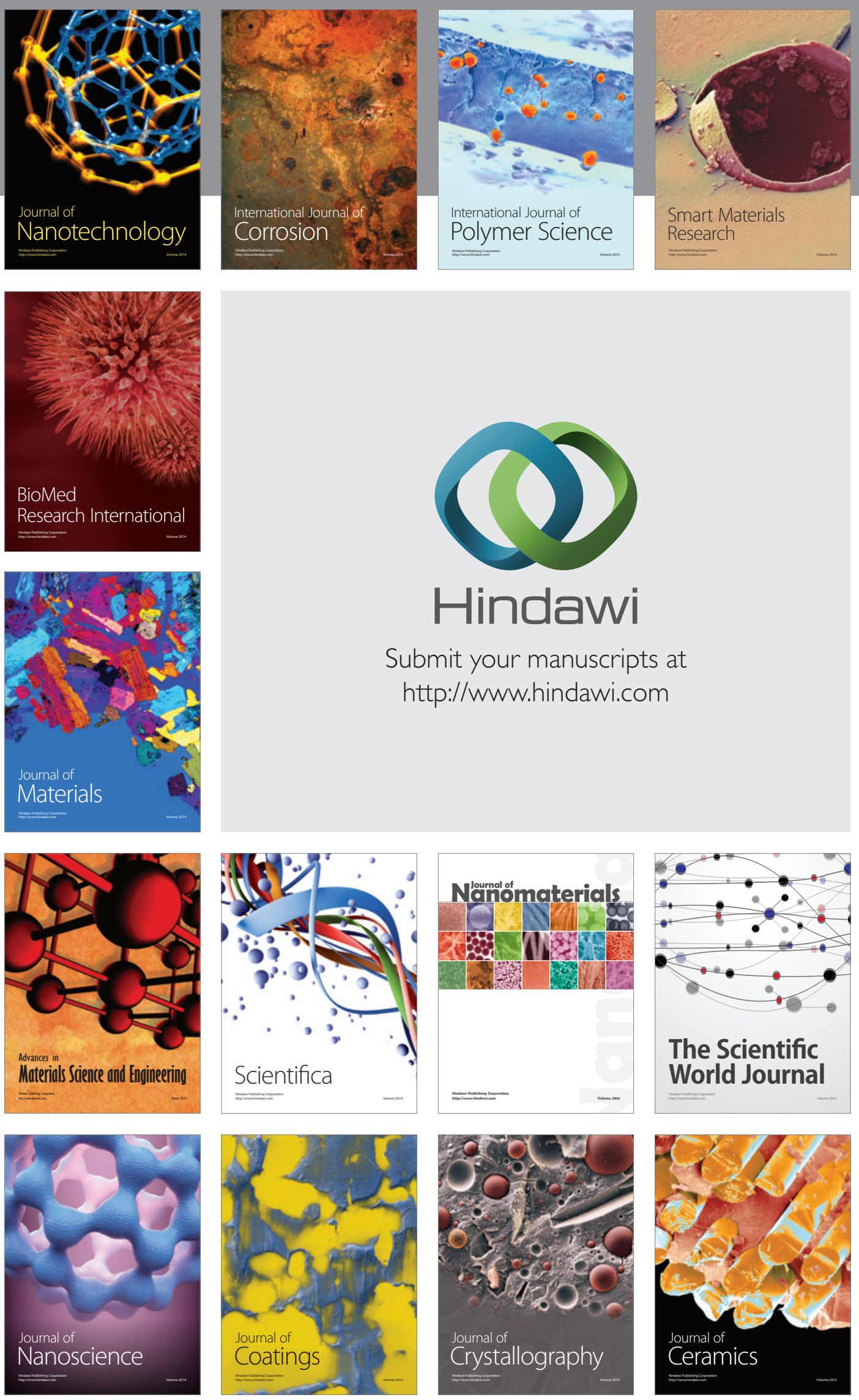

The Scientific World Journal

Submit your manuscripts at

http://www.hindawi.com

\section{World Journal}

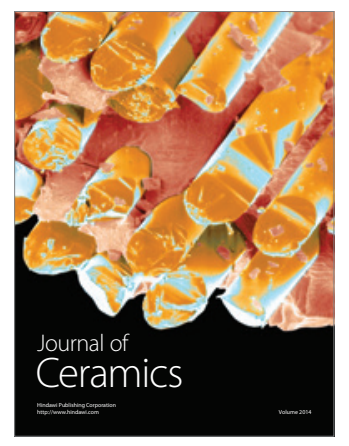

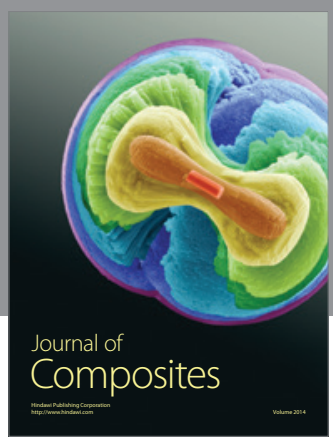
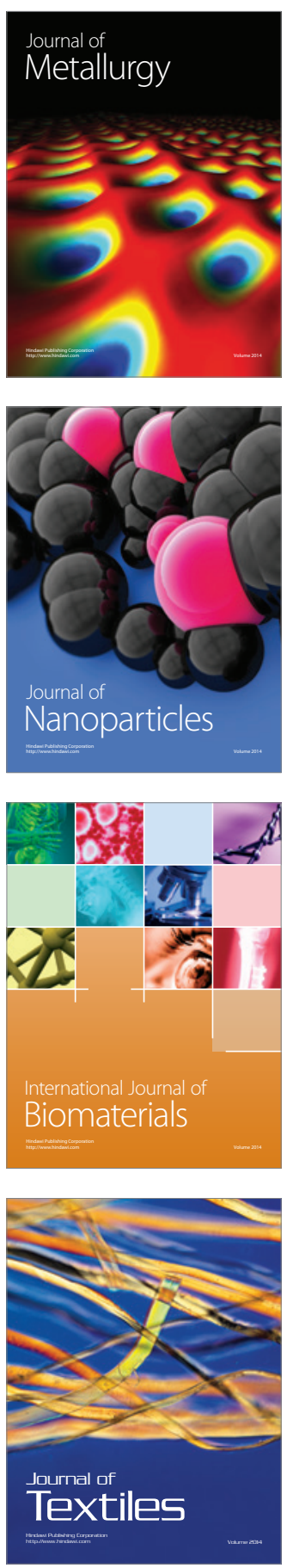